$$
\text { EUI - Series C - } 6.4
$$

Flora (Ed.), Growth to Limits 
European University Institute Institut Universitaire Européen Europäisches Hochschulinstitut Istituto Universitario Europeo

\author{
Series C \\ Political and Social Sciences \\ Sciences Politiques et Sociales \\ Politik- und Sozialwissenschaften \\ Scienze Politiche e Sociali
}

6.4

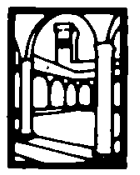

Badia Fiesolana - Firenze 


\title{
Growth to Limits
}

\section{The Western European Welfare States Since World War II}

\author{
Volume 4 \\ Appendix \\ (Synopses, Bibliographies, Tables)
}

Edited by

Peter Flora

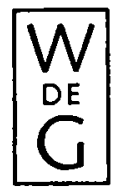

1987

Walter de Gruyter - Berlin - New York 
Library of Congress Cataloging-in-Publication Data (Revised for volume 4)

\section{Growth to limits.}

(Series C--Political and social sciences $=$ Sciences politiques et sociales ; 6 )

Includes bibliographies.

Contents: Sweden, Norway, Finland, Denmark --

v. 2. Germany, United Kingdom, Ireland, Italy.--v. 4.

Appendix (synopses, bibliographies, tables)

1. Europe--Social conditions--20th century. 2. Welfare state.

3. Europe--Politics and government--1945-

4. Europe--Economic and conditions--1945-

I. Flora, Peter, 1944- II. Series: Series C--

Political and social sciences ; 6 .

HN377.G76 $1986 \quad 361.6^{\prime} 5^{\prime} 094 \quad 86-19938$

ISBN 0-89925-266-4 (U.S. : v. 1)

\section{CIP-Kurztitelaufnabme der Deutschen Bibliotbek}

Growth to limits : the Western European welfare states since World War II / ed. by Peter Flora. - Berlin ; New York : de Gruyter (European University Institute : Ser. C, Political and social sciences; 6)

NE: Flora, Peter [Hrsg.]; Istituto Universitario Europeo〈Fiesole): European University Institute / C

Vol. 4. Appendix : (synopses, bibliogr., tab.). - 1987.

ISBN 3-10-011133-0

(C) Copyright 1987 by Walter de Gruyter \& Co., Berlin.

All rights reserved, including those of translation into foreign languages. No part of this book may be reproduced in any form - by photoprint, microfilm, or any other means - nor transmitted nor translated into a machine language without written permission from the publisher.

Dust Cover Design: Rudolf Hübler, Berlin. - Setting: Satz-Rechen-Zentrum, Berlin.

Printing: $\mathrm{H}$. Heenemann GmbH \& Co., Berlin.

Binding: Verlagsbuchbinderei Dieter Mikolai, Berlin.

Printed in Germany. 